\title{
X-ray imaging of human brain tissue down to the molecule level
}

Bert Müller, Anna Khimchenko, Griffin Rodgers, Melissa Osterwalder, Christine Tanner, et al.

Bert Müller, Anna Khimchenko, Griffin Rodgers, Melissa Osterwalder, Christine Tanner, Georg Schulz, "X-ray imaging of human brain tissue down to the molecule level," Proc. SPIE 11886, International Conference on X-Ray Lasers 2020, 1188613 (8 July 2021); doi: 10.1117/12.2592603

SPIE. Event: XVII International Conference on X-Ray Lasers, 2020, Online Only 


\title{
X-ray imaging of human brain tissue down to the molecule level
}

\author{
Bert Müller*a,b, Anna Khimchenko ${ }^{\mathrm{a}}$, Griffin Rodgers ${ }^{\mathrm{a}, \mathrm{b}}$, Melissa Osterwalder ${ }^{\mathrm{a}, \mathrm{b}}$, Christine Tanner $^{\mathrm{a}, \mathrm{b}}$, \\ and Georg Schulz ${ }^{\mathrm{a}-\mathrm{c}}$ \\ aBiomaterials Science Center, Department of Biomedical Engineering, University of Basel, \\ Gewerbestrasse 14, 4123 Allschwil, Switzerland; 'biomaterials Science Center, Department Clinical \\ Research, c/o University Hospital Basel, Schanzenstrasse 55, 4031 Basel, Switzerland; 'Core \\ Facility Micro- and Nanotomography, Department of Biomedical Engineering, \\ University of Basel, Gewerbestrasse 14, 4123 Allschwil, Switzerland
}

\begin{abstract}
$\mathrm{X}$ rays have been used for medical imaging since RöNTGEN's fascinating discovery 125 years ago. The first radiographs of human hands were made public less than a month after his famous paper. The conventional X-ray sources integrated into the CT-machines of today's hospitals still rely on the same physical principles. X-ray imaging has traditionally offered high spatial resolution and low contrast for soft tissues such as the brain. Magnetic resonance imaging is therefore the method of choice for brain imaging in a clinical setting, although for cellular resolution studies it suffers from limited spatial resolution. The gold standard in post mortem brain imaging is histology, which involves fixation, embedding, physical sectioning, staining, and optical microscopy. Currently, section thickness limits isotropic voxel sizes to $20 \mu \mathrm{m}$. Advanced X-ray sources including synchrotron radiation facilities offer complementary modalities such as phase-contrast imaging and spatially resolved small-angle X-ray scattering. We showed that X-ray phase contrast of the human cerebellum with micrometer resolution yields complementary three-dimensional images to magnetic resonance microscopy with even better contrast and spatial resolution. Grating interferometry enabled us to visualize individual Purkinje cells in the nonstained cerebellum. Taking advantage of well-established paraffin embedding, Purkinje cells were visualized within the human cerebellum even with conventional instrumentation. Hard X-ray nano-holotomography allowed for label-free, three-dimensional neuroimaging beyond the optical limit with a spatial resolution below $100 \mathrm{~nm}$. Spatially resolved smallangle X-ray scattering permitted the localization of periodic nanostructures such as myelin sheaths on square-inch brain slices and included the orientational information on the axons. These developments have contributed to the establishment of virtual histology and extended the conventional histology to the third dimension. Further advances are required to image the entire human brain with an isotropic micrometer resolution and to suitably handle the petabyte datasets.
\end{abstract}

Keywords: Computed tomography; synchrotron radiation; small-angle X-ray scattering; biological cell imaging; isotropic three-dimensional imaging; hierarchical imaging; virtual histology; nanotomography

\section{INTRODUCTION}

In the second half of the $19^{\text {th }}$ century, experiments with highly energetic electron beams gave rise to RöNTGEN's fascinating discovery of the $\mathrm{X}$ rays. The first radiographs of human hands were made public 125 years ago [1]. The medical personnel of today's hospitals and dental offices regularly uses hard X-ray radiography and CT mainly for diagnostic purposes. The X-ray sources integrated into these systems still rely on the physical principles RöNTGEN introduced more than 125 years ago.

Research activities in the field of high-resolution hard X-ray imaging, however, often employ synchrotron radiation facilities, which have many advantages compared to conventional systems. They offer unique brilliance, polarization, and pulsed time structure [2]. The combination of the synchrotron radiation facility's photon flux with monochromators has led to tunable X-ray beams with reasonably high intensity, i.e. the photon energy can be selected so that the specimen becomes semi-transparent for optimized absorption-based imaging [3]. Artefacts well known from conventional sources including beam hardening are avoided.

*bert.mueller@unibas.ch; phone 4161 207-5430; www.bmc.unibas.ch 
Despite the numerous advantages, synchrotron radiation facilities have fundamental drawbacks. The imaging experiments have to be carefully planned well in advance, since the usage requires successful beamtime applications. Therefore, liquid metal and inverse Compton scattering X-ray sources $[4,5]$ were introduced, for example, to exploit phase-contrast imaging in the local laboratory environment.

High-resolution imaging experiments, such as phase-contrast imaging and spatially resolved small-angle X-ray scattering, can only be performed on biopsies and human tissue post mortem, because the dose exceeds the medically acceptable limits. Therefore, these X-ray-based techniques are complementary to the ultrasound and magnetic resonancebased in vivo methods with millimeter resolution. Even magnetic resonance microscopy cannot resolve microstructures below about $10 \mu \mathrm{m}$. Figure 1 illustrates the complementary nature of the imaging techniques to visualize the brain down to molecular scale.

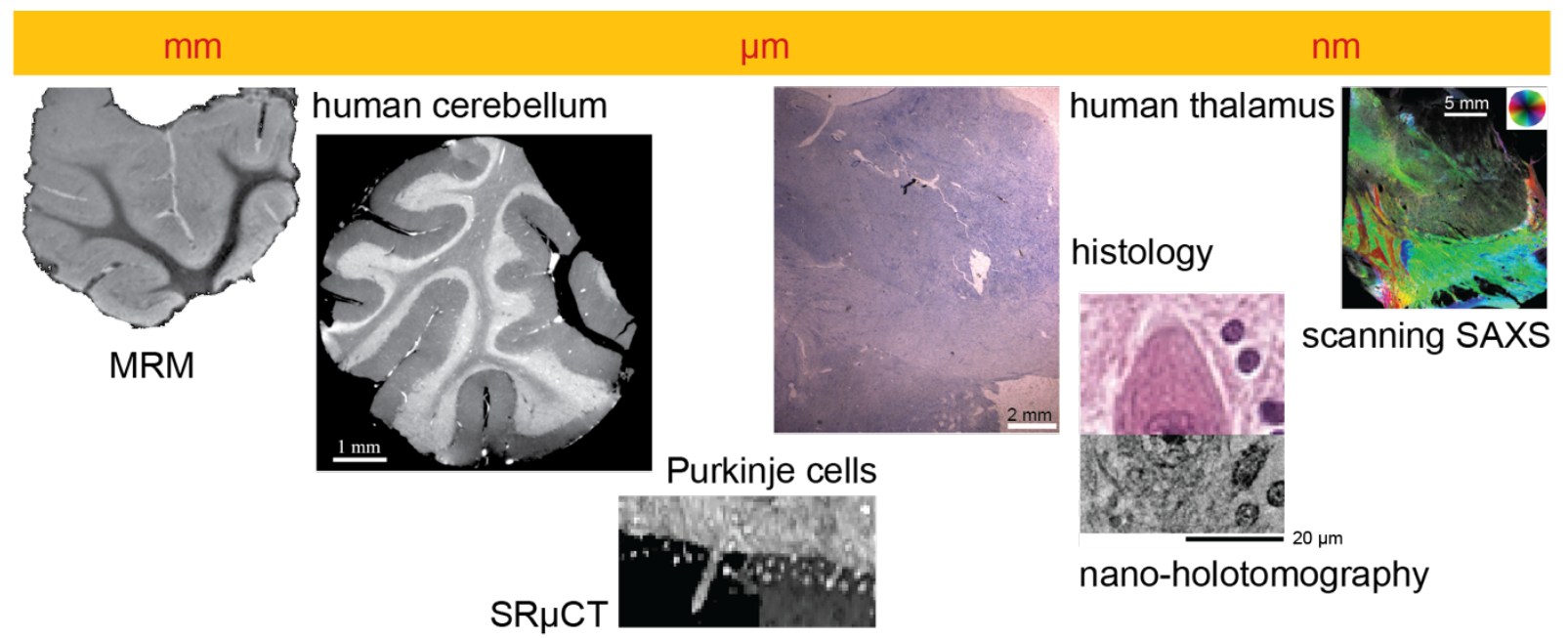

Figure 1. Magnetic resonance microscopy (MRM) is well suited to image brain tissue with voxel sizes of some ten micrometers [6]. Grating-based phase tomography of the human cerebellum provides a different contrast mechanism and true micrometer resolution [6]. It allowed for the three-dimensional imaging of individual Purkinje cells without using contrast agents [7]. The well-established optical microscopy of histological slides complements the tomographic imaging, because the application of dedicated stains enables functional imaging in two-dimensional fashion after tissue slicing. Even without the application of stains, one can make visible the abundance and orientation of periodic nanostructures such as myelin sheaths by spatially resolved small-angle X-ray scattering (SAXS) [8].

\subsection{Gold standard for the visualization of cells in human tissues}

The gold standard for investigating biological tissues at the cellular level is histology. It involves a series of tissue preparation procedures. First, the brain tissue is fixated, for example using formalin solution. Second, the fixated brain tissue is sliced either by sectioning at cryogenic temperatures or after embedding into paraffin. Before optical microscopy is applied, the slices are stained by means of preselected chemicals. This approach is not only established as a part of clinical practice at pathology departments, but also applied for brain research. Despite the two-dimensional nature of histological sections, serial sectioning allows for the three-dimensional visualization of tissues [9]. For example, in 2013 the BigBrain project created an atlas of the full human brain with $20 \mu \mathrm{m}$ isotropic voxels based on the combination of over 7,000 full brain histological sections with a data size of more than 1 TB [10]. The researchers aligned the two-dimensional sections by registration with an MRI volume recorded prior to histology. It should be noted, however, that the process of serial sectioning introduces tissue deformations such as rips, folds, and shears. Furthermore, the staining often introduces modulations in the brightness of the optical micrographs, especially for slides of centimeter size.

The spatial resolution in conventional histology is limited by the slice thickness, precluding the visualization of cellular or subcellular details in three-dimensional space without specialized, time-consuming acquisition protocols. One such approach involves simultaneous sectioning and optical microscopy, allowing for the atlas of an entire mouse brain (GolgiCox-stained and resin-embedded) at voxel sizes of $0.33 \mu \mathrm{m} \times 0.33 \mu \mathrm{m} \times 1.00 \mu \mathrm{m}$ and requiring ten days of continuous data acquisition to yield the roughly 8 TB raw volume [11]. 
Histological sectioning combined with electron microscopy is another approach, but penetration depths below $1 \mu \mathrm{m}$ result in similar limitations related to sectioning. Other alternatives include magnetic resonance imaging, which offers a non-destructive, isotropic visualization and can be applied to larger volumes. Unfortunately, even the best available magnetic resonance imaging facilities only reach pixel sizes of a few tens of micrometers. Confocal microscopy has been used for volumetric imaging of brain tissue with volumes on the order of $4 \mathrm{~mm}^{3}$ [12]. Confocal light sheet microscopy has been employed for the visualization of an entire mouse brain at around $1 \mu \mathrm{m}$ voxel length. This technique, however, relies on tissue clearing and on fluorescent dyes [13], which are usually incompatible with subsequent conventional histology.

\subsection{Phase-contrast imaging using hard $X$ rays}

Hard X rays have a penetration depth to overcome the limitation of sectioning [14] and provide micrometer or even nanometer resolution in tomographic imaging. As brain tissues are composed of low $Z$ elements, conventional X-ray absorption provides hardly any contrast. Therefore, the phase-contrast modalities are preferable, especially for non-stained soft tissues $[15,16]$. The contrast advantage for phase, i.e. the relation between the real and the imaginary parts of the refractive index, is several orders of magnitude, as pointed by the pioneer in the field, U. Bonse, decades ago [17].

Currently, there are several methods for phase retrieval in hard X-ray imaging [15, 16]. A powerful and frequently used method was proposed in 2002 by D. Paganin and coworkers, wherein a FOURIER-space filter is applied to standard radiographic projections to retrieve the phase [18]. This phase retrieval approach is ideal for high-throughput studies, as it allows for the fast and relatively simple data acquisition and processing. The interpretation of the derived quantities must be done with care, as it relies on a reasonable estimate of the ratio between the real to the imaginary part of the refractive index and the assumption that it is nearly constant throughout the specimen [19-21].

\subsection{X-ray-based virtual histology}

X-ray-based virtual histology, a synonym for high-resolution hard X-ray computed tomography of fixated and embedded tissues, has extended conventional histology to the third dimension with an isotropic resolution below $1 \mu \mathrm{m}$ [22]. X-raybased virtual histology with the conventional absorption contrast was applied to bone, as demonstrated by e.g. P. Schneider et al. [23]. X-ray staining protocols have been employed to improve the absorption contrast in X-ray-based virtual histology of soft tissues [24, 25].

For the visualization of the cellular structure of soft tissues without staining, X-ray-based virtual histology has been based on phase contrast. Here, the X-ray-based virtual histology of lungs is a pioneering and early example, as the airtissue interfaces yield an especially strong phase contrast [26]. J. Albers and coworkers [22] recently summarized the wide variety of other X-ray-based virtual histology studies for soft tissues. Synchrotron radiation-based phase-contrast X-raybased virtual histology of lung, brain, and heart tissues shows that the phase contrast imaging provides a quality superior to the conventional approaches involving staining protocols at substantially lower radiation dose [27]. Several research teams have demonstrated phase-contrast X-ray-based virtual histology using advanced laboratory-based systems. For example, M. Töpperwien and coworkers [28] recently published a profound X-ray-based virtual histology study of paraffin-embedded human cerebellum using a liquid-metal jet source. The combination of the tomography data with automatic segmentation algorithms allowed for the three-dimensional localization of more than one million neurons in a volume of about $1 \mathrm{~mm}^{3}$. Compared to the synchrotron facilities, these advanced systems have been available in the laboratory of the researchers throughout the year but yield only limited photon flux even for a broad spectrum. The imaging of large volumes combined with high spatial resolution is, therefore, currently reserved for synchrotron radiation-based virtual histology.

Thanks to the essentially non-destructive nature of hard $X$ rays, the subsequent validation of the imaging results by the established conventional histology is possible. Thus, X-ray-based virtual histology is also applied to select the cutting planes for histological sectioning [29], which has been traditionally done only by optical inspection, i.e. without the knowledge of the tissue features in the bulk. Therefore, X-ray-based virtual histology has been suggested as a valuable addition to the current workflow of histology in clinical and research settings [22].

\subsection{Visualization of larger volumes}

The extension of high-resolution X-ray-based virtual histology to centimeter-size objects is prevented either by the limited photon-beam size and by the restrictions of the detector. Laboratory-based X-ray sources with their cone beam allow for the full-field illumination of objects as large as the entire human skull, but limited flux reduces high-resolution acquisition for large specimens. Synchrotron radiation sources provide orders of magnitude higher photon flux, but the beam cross section is often limited to square millimeters [30-32]. Additionally, currently available detectors have generally 
$4000 \times 4000$ pixel arrays at best, limiting the spatial resolution to about a thousandth of the object's diameter for full-field acquisitions. For example, the field-of-view corresponds only to $4 \mathrm{~mm}$ when using $1 \mu \mathrm{m}$-wide effective pixels.

Local tomography is a technique, where a tomogram is taken from a small part of the specimen [33]. This approach allows for the reconstruction of a part of a specimen, even if the sample is significantly larger than the X-ray beam's cross section and the effective detector width. A series of local tomography reconstructions can be combined to obtain the complete image of a large object [34-37]. Due to the truncated projections taken in local tomography, the resulting reconstructions are prone to artifacts $[36,38,39]$. These artifacts can be reduced by prior knowledge of the specimen geometry, extrapolating data into truncated regions [36], or with specialized reconstruction techniques [39].

An alternative approach is to create a mosaic projection (or sinogram) by stitching many limited field-of-view acquisitions before reconstruction. The field-of-view can be almost doubled by off-axis acquisition, wherein projections taken 180 degrees apart are combined prior to reconstruction $[36,40]$. Even for phase-contrast imaging, this off-axis acquisition techniques allows for increased field-of-view without reducing contrast or spatial resolution [41]. Additionally, stitching radiographs enabled us to image objects, which are more than $30 \mathrm{~mm}$ in diameter such as cochlear implants and the surrounding tissues including bone [42]. This approach requires increased effort, since not only the sinograms are larger, but also the number of projections has to be raised. The limits of stitching techniques are currently a matter of discussion $[34,36]$. Some research teams use the term tomosaic for techniques involving stitching many fields-of-view [34]. On the one hand, this approach requires significant computational resources and precise translational stages. On the other hand, it provides a dose-efficient acquisition compared to recording numerous local tomography scans [34]. Recently, this approach has allowed for the imaging of a complete mouse brain with $0.8 \mu \mathrm{m}$-wide pixels. It has required the stitching of a mosaic grid of $12 \times 11$ images per projection and a dedicated data analysis package for handling the tera-voxel dataset [34]. With a volume of one cubic centimeter, this measurement is around three orders of magnitude larger than previously performed studies. A complete human brain atlas, however, requires a 3,000 times larger volume. Consequently, dedicated protocols for the specimen preparation as well as the data acquisition and processing have to be further developed.

\subsection{Current $\mathrm{X}$-ray-based initiatives for high-resolution brain imaging}

Currently, there are several large-scale research initiatives across the globe dedicated to brain research, including major efforts in Europe, the US, Canada, China, Korea, Taiwan, Japan, and Australia [43]. Brain imaging and brain atlases play a dominant role in these projects, as they provide information on the microanatomy of the brain. For example, the MultiLevel Human Brain Atlas is a co-design project of the Human Brain Project, a Flagship project of the European Union, which looks to build a multimodal, hierarchical brain atlas [44]. Public availability and data sharing including high-quality brain atlases are essential for these initiatives [43]. Therefore, most recently, the Taiwanese synchrotron-radiation facility proposed a dedicated beamline, denoted 02A, which will be exclusively used for X-ray imaging for brain science. This beamline will be realized during Phase-II of that synchrotron radiation facility [45]. At the European Synchrotron Radiation Facility (ESRF), the flagship beamline BM18 is under construction and will be operational during 2021. This beamline will have a huge beam diameter and will allow for an automated hierarchical imaging of entire human organs.

\section{TOWARDS X-RAY IMAGING OF THE ENTIRE HUMAN BRAIN}

\subsection{Magnetic resonance imaging for the determination of formalin-induced deformations in human brain}

There is mutual consensus that hard X-ray computed tomography (CT) with true micrometer resolution should not be applied to living species especially humans, because serious radiation damage is expected, often resulting in diseases including cancer. Therefore, high-resolution hard X-ray studies of human tissues are performed post mortem. Nevertheless, the organ's anatomy should be visualized as close as possible to the in vivo conditions. Therefore, the human brain was investigated post mortem by means of clinical magnetic resonance imaging (MRI). First, images with the brain inside the skull of the dead body were acquired. After removal of the brain from the skull, it swelled by about 5\% [46]. Subsequent to placing the human brain into formalin solution, a dozen MRI datasets during the formalin penetration were recorded, which led to local compressive and tensile strains larger than $\pm 20 \%$. The non-rigid registration of the MRI datasets allowed for the precise quantification of the entire formalin-induced deformation fields [46]. This knowledge enables computer-based correction of high-resolution CT-data by less detailed MRI-data.

\subsection{Imaging biological cells using hard $X$-ray tomography}

A. Lareida et al. discovered how to visualize osmium-stained ganglion cells present in the organ of Corti [47]. The nondestructive neuron cell counting in a selected volume of $125 \mu \mathrm{m} \times 800 \mu \mathrm{m} \times 600 \mu \mathrm{m}$ gave rise to the presence of 2,000 
ganglion cells along one millimeter of the organ of Corti. Therefore, the study demonstrated for the first time that hard Xray tomography allows visualizing individual cells within human nerve tissue. Although ganglion cells are encapsulated in highly X-ray absorbing bony tissue, individual ganglion cells were counted and measured in size and shape without any sectioning and related artefacts. Consequently, the paper provided an important basis for the direct comparison of healthy and altered inner ear morphologies such as dysplastic malformations down to the sub-cellular level.

Already a year later, G. Schulz et al. visualized individual Purkinje cells in non-stained human cerebellum [7]. The uniqueness of the grating-based hard X-ray-based phase-contrast tomographic imaging became clear by the detailed comparison with MR microscopy and selected histological sections of the same part of the human brain [6]. For such a comparison, one needs powerful registration algorithms [48]. N. Chicherova et al. performed dedicated research activities for the non-rigid slice-to-volume registration as required for the identification of the counterparts of histology slides within CT-data [49-51]. This task is particularly challenging due to the many degrees of freedom [52]. The registration of twodimensional histology slides to X-ray-based virtual histology volumes allows for the validated interpretation of tomography results and for the colorization of tomography datasets according to the well-known stains in histology, i.e. to extend the histology into the third dimension [53]. This study was mainly based on advanced laboratory CT and demonstrated the power of paraffin embedding in brain imaging. A. Khimchenko et al. have shown for the first time that non-stained cells in human brain tissue can be made visible even with conventional high-resolution CT-systems [53]. Nevertheless, at synchrotron radiation facilities the resolution of tomography systems can be pushed to a fraction of a micrometer and subcellular structures are clearly detectable [54] thanks to the superior density resolution of phase-contrast mode compared with absorption mode. The phase tomography approaches, i.e. grating-based interferometry, holotomography, and single-distance phase retrieval were experimentally compared [19, 20]. These studies, performed on soft tissues of mice and rats, showed that single-distance phase retrieval minimizes the necessary time for data acquisition and the size of raw data to be recorded. Therefore, single-distance phase retrieval should be considered for future projects on three-dimensional imaging of human brain with hard $\mathrm{X}$ rays.

\section{HARD X-RAY-BASED NANOTOMOGRAPHY OF HUMAN BRAIN TISSUE}

\subsection{Nanoholotomography at synchrotron radiation facilities}

Although X-ray-based virtual histology of paraffin-embedded brain tissue based on synchrotron radiation has allowed for the visualization of subcellular details and automated cell counting [54], the application of X-ray optics enabled tomographic imaging beyond the optical limit, as recently demonstrated [55]. Nanoholotomography has pushed X-raybased virtual histology beyond the 100-nm limit, with isotropic voxel sizes down to $25 \mathrm{~nm}$ and the ability to study the anatomical features of individual cells, e.g. organelles, in brain tissues by hard X rays [55]. This approach is unique because not only is spatial resolution superior to optical microscopy, but also the accessible volumes are substantially larger than in electron-microscopy based techniques. Therefore, the gap between conventional histology and electron-based methods has been bridged.

\subsection{Nanoholotomography of human cortex}

Video 1 demonstrates the potential and the performance of nanoholotomography. The three-dimensional images originated from a part of the human cortex after embedding into paraffin [55]. The individual slices resemble electron microscopy images. The tomography data, however, are three-dimensional consisting of cubic voxels with a length of $50 \mathrm{~nm}-$ this means each virtual slice is only $50 \mathrm{~nm}$ thin. Video 1 corresponds to the scroll mode through this cylinder with a maximal diameter of $510 \mu \mathrm{m}$. First of all, one recognizes the capillary system for the blood supply. But one can also easily see the cell layers containing the neurons. These cells can be segmented to determine their density and morphology [55]. The spatial and density resolution of the tomography data, however, even allows for the identification of sub-cellular structures. Nanometer-size anatomical features, such as cell soma, nuclear envelope, nuclear pore, nucleolus, and dendrite, are distinguishable [55]. 


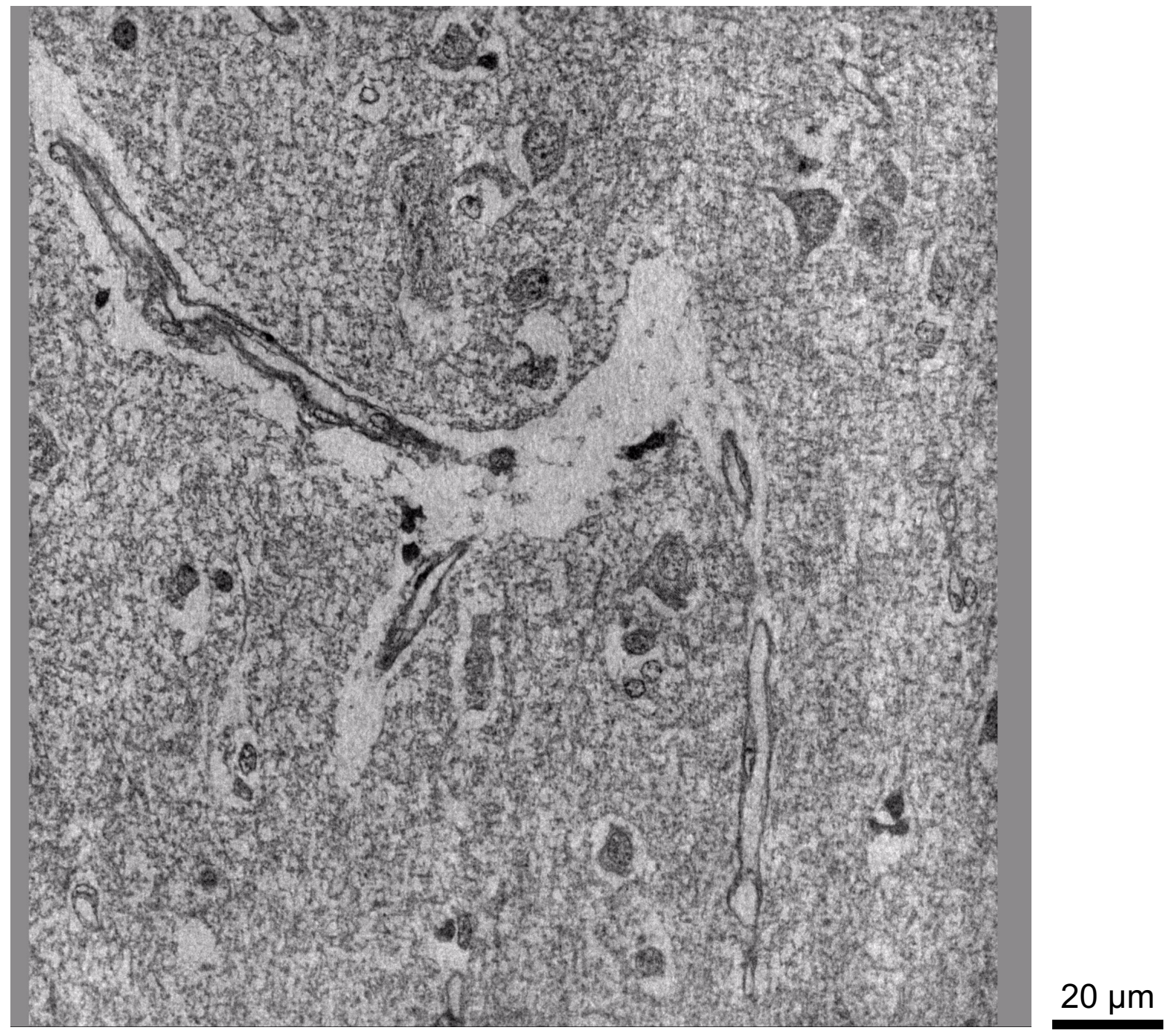

Video 1 . A set of $50 \mathrm{~nm}$-thin virtual slices from nanotomography data of the human neocortex after formalin fixation and paraffin embedding. Besides the blood vessels, one can clearly identify subcellular structures, which include the cell somas, the nuclear envelopes, the nuclear pores, the nucleoli, and the dendrites of the cells present (astrocytes, small pyramidal and numerous stellate neurons, medium-size pyramidal and non-pyramidal neurons with vertically oriented intracortical axons, and other types of stellate and pyramidal neurons). http://dx.doi.org/10.1117/12.2592603.1

\section{SPATIALLY RESOLVED SMALL-ANGLE X-RAY SCATTERING FROM A NON- STAINED SLIDE OF HUMAN BRAIN}

\subsection{Conventional histology for myelin imaging}

In order to localize the myelin with micrometer precision and to determine its abundance, one can prepare a cryostat section with a thickness of about $50 \mu \mathrm{m}$ [56]. This thin section has to be appropriately stained for myelin [57], before an optical micrograph can be recorded, see for example Schulz et al. [8].

\subsection{X-ray scattering for myelin imaging}

As a valid alternative, one can take advantage of spatially resolved small-angle X-ray scattering to circumvent the staining [58]. The scattering signal is observed at angles, which inversely correspond to the nanostructure's size. The myelin sheaths responsible for insulating axons exhibit a characteristic periodicity between 15 and $18 \mathrm{~nm}$ [8]. Therefore, related rings were found on pattern of the two-dimensional detector. These rings exhibit a modulation in intensity associated with the 
orientation of the myelin sheaths and the surrounded axons [8]. These two-dimensional images are complementary to the conventional histology data because they not only represent the abundance of myelin but also include the orientation of the myelin sheaths. Consequently, the orientation of the axons can be determined on brain slices several square-inches in size. The approach can even be extended to slices of the entire human brain in health and disease.

\section{ACKNOWLEDGEMENT}

The authors gratefully acknowledge the financial support of the Swiss National Science Foundation (Project No. 185058). The authors also acknowledge the work of staff at the European Synchrotron Radiation Facility, Grenoble, France. The data were collected during the approved beamtime MD-969.

\section{REFERENCES}

[1] Röntgen, W. C., Haase, A., Landwehr, G., and Umbach, E., [Röntgen centennial: X-rays in natural and life sciences] World Scientific, Singapore; River Edge, NJ(1997).

[2] Wilmott, P., [Introduction to synchrotron radiation: Techniques and applications] John Wiley \& Sons, (2019).

[3] Grodzins, L., "Optimum energies for x-ray transmission tomography of small samples: Applications of synchrotron radiation to computerized tomography I," Nuclear Instruments and Methods in Physics Research 206(3), 541-545 (1983).

[4] Hornberger, B., Kasahara, J., Ruth, R., Loewen, R., and Khaydarov, J., "Inverse Compton scattering X-ray source for research, industry and medical applications," Proc. SPIE, (2021).

[5] Hertz, H. M., "High-resolution biomedical imaging with laboratory x-ray sources," Proc. SPIE, (2021).

[6] Schulz, G., Waschkies, C., Pfeiffer, F., Zanette, I., Weitkamp, T., David, C., and Müller, B., "Multimodal imaging of human cerebellum - merging X-ray phase microtomography, magnetic resonance microscopy and histology," Scientific Reports 2, (2012).

[7] Schulz, G., Weitkamp, T., Zanette, I., Pfeiffer, F., Beckmann, F., David, C., Rutishauser, S., Reznikova, E., and Müller, B., "High-resolution tomographic imaging of a human cerebellum: comparison of absorption and grating-based phase contrast," Journal of The Royal Society Interface, rsif20100281 (2010).

[8] Schulz, G., Deyhle, H., Bikis, C., Bunk, O., and Müller, B., "Imaging the orientation of myelin sheaths in a nonstained histological slice of human brain," Precision Nanomedicine 3(4), 656-665 (2020).

[9] Pichat, J., Iglesias, J. E., Yousry, T., Ourselin, S., and Modat, M., "A Survey of Methods for 3D Histology Reconstruction," Medical Image Analysis 46, 73-105 (2018).

[10] Amunts, K., Lepage, C., Borgeat, L., Mohlberg, H., Dickscheid, T., Rousseau, M.-É., Bludau, S., Bazin, P.-L., Lewis, L. B., and Oros-Peusquens, A.-M., "BigBrain: an ultrahigh-resolution 3D human brain model," Science 340(6139), 1472-1475 (2013).

[11] Li, A., Gong, H., Zhang, B., Wang, Q., Yan, C., Wu, J., Liu, Q., Zeng, S., and Luo, Q., "Micro-optical sectioning tomography to obtain a high-resolution atlas of the mouse brain," Science 330(6009), 1404-1408 (2010).

[12] Sands, G. B., Gerneke, D. A., Hooks, D. A., Green, C. R., Smaill, B. H., and Legrice, I. J., "Automated imaging of extended tissue volumes using confocal microscopy," Microscopy research and technique 67(5), 227-239 (2005).

[13] Silvestri, L., Bria, A., Sacconi, L., Iannello, G., and Pavone, F. S., "Confocal light sheet microscopy: micronscale neuroanatomy of the entire mouse brain," Optics Express 20(18), 20582-20598 (2012).

[14] Henke, B. L., Gullikson, E. M., and Davis, J. C., "X-ray interactions: photoabsorption, scattering, transmission and reflection $\mathrm{E}=50-30,000 \mathrm{eV}, \mathrm{Z}=1-92, "$ (1993). 
[15] Momose, A., "Recent advances in X-ray phase imaging," Japanese Journal of Applied Physics 44(9R), 6355 (2005).

[16] Fitzgerald, R., "Phase-sensitive x-ray imaging," Phys. Today 53(7), 23-26 (2000).

[17] Bonse, U., "Röntgen-Mikrotomographie," Physikalische Blätter 53(3), 211-214 (1997).

[18] Paganin, D., Mayo, S., Gureyev, T. E., Miller, P. R., and Wilkins, S. W., "Simultaneous phase and amplitude extraction from a single defocused image of a homogeneous object," Journal of microscopy 206(1), 33-40 (2002).

[19] Zanette, I., Lang, S., Rack, A., Dominietto, M., Langer, M., Pfeiffer, F., Weitkamp, T., and Müller, B., "Holotomography versus X-ray grating interferometry: A comparative study," Applied Physics Letters 103(24), 244105 (2013).

[20] Lang, S., Zanette, I., Dominietto, M., Langer, M., Rack, A., Schulz, G., Le Duc, G., David, C., Mohr, J., and Pfeiffer, F., "Experimental comparison of grating-and propagation-based hard X-ray phase tomography of soft tissue," Journal of Applied Physics 116(15), 154903 (2014).

[21] Gureyev, T., Mohammadi, S., Nesterets, Y., Dullin, C., and Tromba, G., "Accuracy and precision of reconstruction of complex refractive index in near-field single-distance propagation-based phase-contrast tomography," Journal of Applied Physics 114(14), 144906 (2013).

[22] Albers, J., Pacilé, S., Markus, M., Wiart, M., Velde, G. V., Tromba, G., and Dullin, C., "X-ray-Based 3D Virtual Histology_Adding the Next Dimension to Histological Analysis," Molecular Imaging and Biology, 110 (2018).

[23] Schneider, P., Meier, M., Wepf, R., and Müller, R., "Towards quantitative 3D imaging of the osteocyte lacunocanalicular network," Bone 47(5), 848-858 (2010).

[24] Metscher, B. D., "MicroCT for comparative morphology: simple staining methods allow high-contrast 3D imaging of diverse non-mineralized animal tissues," BMC Physiology 9(1), 11 (2009).

[25] Dullin, C., Ufartes, R., Larsson, E., Martin, S., Lazzarini, M., Tromba, G., Missbach-Guentner, J., PinkertLeetsch, D., Katschinski, D. M., and Alves, F., " $\mu$ CT of ex-vivo stained mouse hearts and embryos enables a precise match between 3D virtual histology, classical histology and immunochemistry," PLOS ONE 12(2), e0170597 (2017).

[26] Kitchen, M. J., Paganin, D., Lewis, R. A., Yagi, N., Uesugi, K., and Mudie, S. T., "On the origin of speckle in x-ray phase contrast images of lung tissue," Physics in Medicine \& Biology 49(18), 4335 (2004).

[27] Saccomano, M., Albers, J., Tromba, G., Dobrivojević Radmilović, M., Gajović, S., Alves, F., and Dullin, C., "Synchrotron inline phase contrast $\mu \mathrm{CT}$ enables detailed virtual histology of embedded soft-tissue samples with and without staining," Journal of Synchrotron Radiation, (2018).

[28] Töpperwien, M., van der Meer, F., Stadelmann, C., and Salditt, T., "Three-dimensional virtual histology of human cerebellum by X-ray phase-contrast tomography," Proceedings of the National Academy of Sciences, 201801678 (2018).

[29] Stalder, A., Ilgenstein, B., Chicherova, N., Deyhle, H., Beckmann, F., Müller, B., and Hieber, S. E., "Combined use of micro computed tomography and histology to evaluate the regenerative capacity of bone grafting materials," International Journal of Materials Research 105(7), 679-691 (2014).

[30] Weitkamp, T., Tafforeau, P., Boller, E., Cloetens, P., Valade, J. P., Bernard, P., Peyrin, F., Ludwig, W., Helfen, L., and Baruchel, J., "Status and evolution of the ESRF beamline ID19," AIP Conference Proceedings 1221(1), 33-38 (2010).

[31] Pešić, Z. D., Fanis, A. D., Wagner, U., and Rau, C., "Experimental stations at I13 beamline at Diamond Light Source," Journal of Physics: Conference Series 425(18), 182003 (2013).

[32] Haibel, A., Ogurreck, M., Beckmann, F., Dose, T., Wilde, F., Herzen, J., Müller, M., Schreyer, A., Nazmov, V., Simon, M., Last, A., and Mohr, J., "Micro-and nano-tomography at the GKSS Imaging Beamline at PETRA III." 7804, 8. 
[33] Kuchment, P., Lancaster, K., and Mogilevskaya, L., "On local tomography," Inverse Problems 11(3), 571 (1995).

[34] Vescovi, R., Du, M., Andrade, V. d., Scullin, W., Gürsoy, D., and Jacobsen, C., "Tomosaic: efficient acquisition and reconstruction of teravoxel tomography data using limited-size synchrotron X-ray beams," Journal of synchrotron radiation 25(5), (2018).

[35] Oikonomidis, I. V., Lovric, G., Cremona, T. P., Arcadu, F., Patera, A., Schittny, J. C., and Stampanoni, M., "Imaging samples larger than the field of view: the SLS experience." 849, 012004.

[36] Kyrieleis, A., Titarenko, V., Ibison, M., Connolley, T., and Withers, P., "Region-of-interest tomography using filtered backprojection: assessing the practical limits," Journal of microscopy 241(1), 69-82 (2011).

[37] Haberthür, D., Hintermüller, C., Marone, F., Schittny, J. C., and Stampanoni, M., "Radiation dose optimized lateral expansion of the field of view in synchrotron radiation X-ray tomographic microscopy," Journal of synchrotron radiation 17(5), 590-599 (2010).

[38] Rashed, E. A., and Kudo, H., "Towards high-resolution synchrotron radiation imaging with statistical iterative reconstruction," Journal of synchrotron radiation 20(1), 116-124 (2013).

[39] Kudo, H., Suzuki, T., and Rashed, E. A., "Image reconstruction for sparse-view CT and interior CTintroduction to compressed sensing and differentiated backprojection," Quantitative imaging in medicine and surgery 3(3), 147 (2013).

[40] Stock, S. R., "Recent advances in X-ray microtomography applied to materials," International Materials Reviews 53(3), 129-181 (2008).

[41] Schulz, G., Weitkamp, T., Zanette, I., Pfeiffer, F., Müller-Gerbl, M., David, C., and Müller, B., "Asymmetric rotational axis reconstruction of grating-based x-ray phase contrast tomography of the human cerebellum." 8506, 10.

[42] Müller, B., Bernhardt, R., Weitkamp, T., Beckmann, F., Bräuer, R., Schurigt, U., Schrott-Fischer, A., Glueckert, R., Ney, M., Beleites, T., Jolly, C., and Scharnweber, D., "Morphology of bony tissues and implants uncovered by high-resolution tomographic imaging," International Journal of Materials Research 98(7), 613-621 (2007).

[43] Huang, Z. J., and Luo, L., "It takes the world to understand the brain," Science 350(6256), $42-44$ (2015).

[44] Amunts, K., Ebell, C., Muller, J., Telefont, M., Knoll, A., and Lippert, T., "The Human Brain Project: Creating a European Research Infrastructure to Decode the Human Brain," Neuron 92(3), 574-581 (2016).

[45] Chang, S.-H., Chang, C.-Y., Liu, C.-Y., Chang, C.-F., Chiang, L.-C., Liao, B.-Y., Chen, Y.-S., Chen, H.-H., Yang, S.-M., Lee, T.-T., Chen, Y.-J., Chen, F.-L., and Hwu, Y.-K., "Design of an X-ray 3D microtomography beamline for full-field hard x-ray projection imaging at the Taiwan photon source," AIP Conference Proceedings 2054(1), 060042 (2019).

[46] Schulz, G., Crooijmans, H., Germann, M., Scheffler, K., Müller-Gerbl, M., and Müller, B., "Three-dimensional strain fields in human brain resulting from formalin fixation," Journal of Neuroscience Methods 202(1), 17-27 (2011).

[47] Lareida, A., Beckmann, F., Schrott-Fischer, A., Glueckert, R., Freysinger, W., and Müller, B., "High-resolution $\mathrm{X}$-ray tomography of the human inner ear: synchrotron radiation-based study of nerve fibre bundles, membranes and ganglion cells," Journal of microscopy 234(1), 95-102 (2009).

[48] Müller, B., Deyhle, H., Lang, S., Schulz, G., Bormann, T., Fierz, F. C., and Hieber, S. E., "Three-dimensional registration of tomography data for quantification in biomaterials science," International journal of materials research 103(2), 242-249 (2012).

[49] Chicherova, N., Fundana, K., Müller, B., and Cattin, P. C., "Histology to $\mu$ CT Data Matching Using Landmarks and a Density Biased RANSAC," Medical Image Computing and Computer-Assisted Intervention - MICCAI 2014. 243-250.

[50] Chicherova, N., Hieber, S., Schulz, G., Khimchenko, A., Bikis, C., Cattin, P., and Müller, B., "Automatic histology registration in application to X-ray modalities," Proceedings of SPIE 9967, 996708 (2016). 
[51] Chicherova, N., Hieber, S. E., Khimchenko, A., Bikis, C., Müller, B., and Cattin, P., "Automatic deformable registration of histological slides to $\mu \mathrm{CT}$ volume data," Journal of microscopy 271(1), 49-61 (2018).

[52] Ferrante, E., and Paragios, N., "Slice-to-volume medical image registration: A survey," Medical Image Analysis 39, 101-123 (2017).

[53] Khimchenko, A., Deyhle, H., Schulz, G., Schweighauser, G., Hench, J., Chicherova, N., Bikis, C., Hieber, S. E., and Müller, B., "Extending two-dimensional histology into the third dimension through conventional micro computed tomography," NeuroImage 139, 26-36 (2016).

[54] Hieber, S. E., Bikis, C., Khimchenko, A., Schweighauser, G., Hench, J., Chicherova, N., Schulz, G., and Müller, B., "Tomographic brain imaging with nucleolar detail and automatic cell counting," Scientific reports 6, 32156 (2016).

[55] Khimchenko, A., Bikis, C., Pacureanu, A., Hieber, S. E., Thalmann, P., Deyhle, H., Schweighauser, G., Hench, J., Frank, S., and Müller-Gerbl, M., "Hard X-Ray Nanoholotomography: Large-Scale, Label-Free, 3D Neuroimaging beyond Optical Limit," Advanced Science, 1700694 (2018).

[56] Niemann, K., Mennicken, V. R., Jeanmonod, D., and Morel, A., "The Morel stereotactic atlas of the human thalamus: atlas-to-MR registration of internally consistent canonical model," Neuroimage 12(6), 601-16 (2000).

[57] Schmued, L., and Slikker, W., "Black-Gold: a simple, high-resolution histochemical label for normal and pathological myelin in brain tissue sections," Brain Research 837(1), 289-297 (1999).

[58] Müller, B., Deyhle, H., Bradley, D., Farquharson, M., Schulz, G., Müller-Gerbl, M., and Bunk, O., "Scanning xray scattering: Evaluating the nanostructure of human tissues," European Journal of Nanomedicine 3(1), 30-33 (2010). 